\title{
Elderly patient with atypical leiomyoma of the bladder presenting as flank pain: A case report
}

\author{
Mojtaba Ameli, Mina Rahmandoost \\ Gonabad University of Medical Sciences, Gonabad, Iran.
}

\begin{abstract}
Summary Atypical leiomyoma is a rare tumor of the bladder whose correct diagnosis with imaging techniques and cystoscopy is difficult. This tumor is prevalent in females and more common in middle age. In the present study we report a rare case of atypical leiomyoma presenting as flank pain and history of recurrent urinary tract infections in an elderly female. Ultrasound (US) showed that the wall of bladder was thickening and irregular, especially in the lower part of the bladder. US revealed hypoechoic solid mass with dimensions of $37 \times 26 \mathrm{~mm}$ in the posterior bladder wall protruding into the bladder.

Computed Tomography scan of the patient showed a mass with dimensions of $29 \times 38 \mathrm{~mm}$ in the posterior wall of the bladder that infiltrated the mesenteric fat and also seemed to be invading the intestinal wall.

According to the general condition and age of our patient, we removed all of the mass under spinal anesthesia by transurethral bladder resection (TURBT). Biopsy results showed atypical leiomyoma. About 6 months after the patient follow-up, no recurrence was observed and symptoms had completely resolved.

According to the non-specificity of the imaging, of the age of presentation and of clinical manifestations of atypical leiomyoma differential diagnosis for bladder cancer it is recommended. Only with histopathologic findings, the diagnosis can be confirmed.
\end{abstract}

KEY WORDS: Leiomyoma; Bladder tumor; Elderly.

Submitted 2 September 2017; Accepted 23 September 2017

\section{INTRODUCTION}

The leiomyoma is a rare and benign tumor of the bladder including $0.43 \%$ of all the bladder tumors according to a study. However, it is the most common benign tumor of the bladder (1). It is more prevalent in women and is frequently observed in the fourth and the fifth decades of the life. Well-known atypical leiomyoma arises in the myometrium and it is identified by pleomorphic nuclei, degenerative chromatin and nucleic inclusion. Depending on the tumor size and location it has different symptoms (2). The leiomyoma can occur with urinary obstructive and irritative symptoms (3).

The urinary obstructive symptoms are the most common symptoms of leiomyoma (49\%) whereas about 38\% of the patients have urinary irritative symptoms, $11 \%$ have hematuria and about $19 \%$ are asymptomatic (4). Based on our information an atypical leiomyoma of the bladder in a 75 years old woman complaining of flank pain is a uncommon condition.

\section{Case report}

We present the case of a 75 years old woman with left flank pain for a month. She had a colicky pain which was positional and radiated to hypogastric area. The patient complained of frequency and had a history of frequent urinary tract inflammation but denied to have chills, fever and hematuria. There was no history of surgery and hospital admission in the patient's past medical history but she had a history of hypertension and was taking Losartan and Triamterene by years. There was a soft and without tenderness abdomen with palpable bladder at abdominal examination with no other important finding. Urinary tract inflammation was ruled out by urine analysis. Other laboratory findings were blood urea nitrogen (BUN) $22 \mathrm{mg} / \mathrm{dl}$, serum creatinine $2 \mathrm{mg} / \mathrm{dl}$, white blood count (WBC) 7800 with 75\% neutrophils. At ultrasonography the bladder wall was seen as hypertrophied and irregular especially in the inferior part and a solid hypoechoic mass with $37 \times 26 \mathrm{~mm}$ size was seen on the posterior wall of the bladder protruding into the bladder. At computed tomography (CT) scan it was demonstrated an image of a 29 × $38 \mathrm{~mm}$ mass on the posterior wall of the bladder (Figure 1) infiltrating to the mesenteric fat which seemed to invade the intestinal wall whereas no evidence of obstruction or hepatic metastasis was seen.

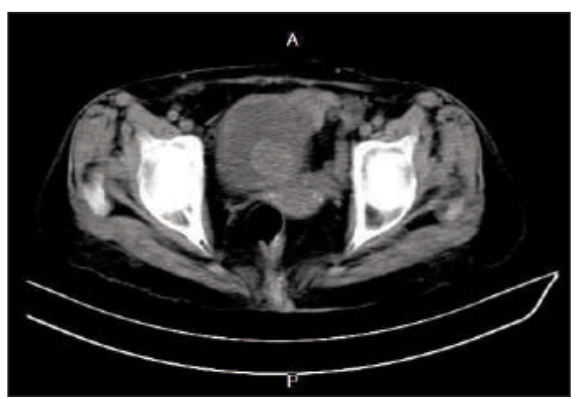

Figure 1.

A mass in bladder in CT

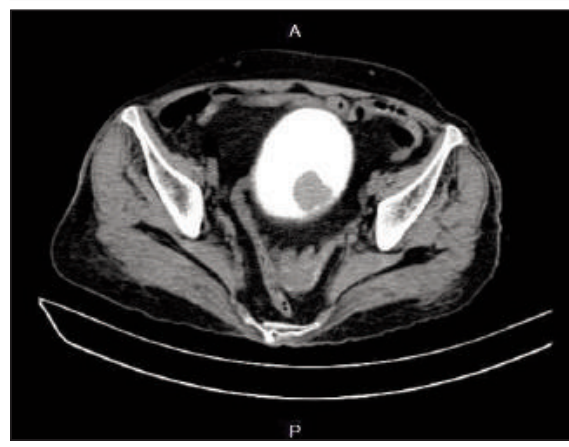
scan without and with contrast. 


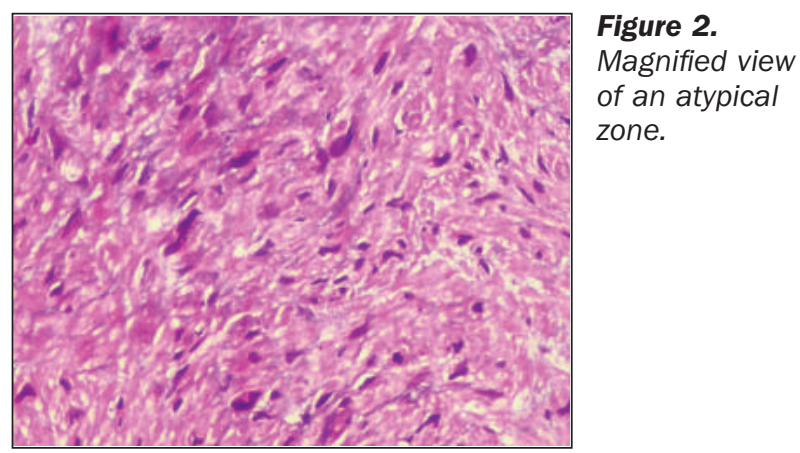

After necessary pre-operative investigations the patient was taken to the operation room where underwent transurethral resection of the bladder of a $4 \mathrm{~cm}$ mass. The histological examination demonstrated stretched and spindle tumor cells with moderately atypical nuclei with sporadic mitosis and no necrosis, therefore atypical leiomyoma was diagnosed (Figure 2).

\section{Discussion}

Leiomyoma was first described by Virchow (4) and bladder leiomyoma is the most common benign tumor of the bladder (1). The bladder leiomyoma can be intravesical, intramural or extra vesical at macroscopic view. Among these three types, the intravesical form is more frequently the cause of bleeding and of irritative and obstructive symptoms as long as they grow into the bladder lumen (5). In the present study, the case of a 75 years old woman is reported who had an uncommon age for the onset of the bladder leiomyoma and at our knowledge is one of the few cases of bladder leiomyoma reported in patients older than 70 . The patient was observed with the uncommon presentation of flank pain that was the unique feature of presentation. The atypical leiomyoma is well known to occur in the myometrium and is characterized by pleomorphic nuclei, degenerative chromatin or nucleic inclusions. Based on our knowledge the atypical leiomyoma is reported in a few cases that were mostly in the childbearing age. Steroidal hormones may have a main role in the development of bladder leiomyoma similarly to what observed in the uterine leiomyoma.

The incidence of an atypical leiomyoma in a 75 years old woman in the menopause age who was not taking any steroidal hormones seems to be controversial and interesting. Ultrasonography, CT scan, magnetic resonance imaging (MRI) and cystoscopy are high value diagnostic tools which can show the morphology and the anatomical location of the leiomyoma. Ultrasound shows leiomyoma as a solid flat hypoechoic tumor with hyperechoic mucosal lining (6). Also CT scan and the MRI are useful for the assessment of the bladder tumors. At CT scan the typical leiomyoma of the bladder is generally described as a round hypodense mass with defined borders and after contrast demonstrates an homogeneous centripetal hyperdensity (7).

The atypical leiomyoma of the bladder may show similar morphology of the bladder cancer and makes difficult the diagnosis with ultrasound, CT, MRI and cystoscopy. The final diagnosis of the bladder lesion can be achieved by histopathological assessment of the biopsy (8). In our case the diagnosis remained uncertain in relation to the patient's age and the tumor aspect at CT scan showing a suspect of intestinal invasion until the definitive diagnosis was made by the histopathological assessment.

The treatment and the prognosis is determined by the size and the location of the tumor. Small asymptomatic tumors may be treated conservatively till onset of the symptoms. For small symptomatic tumors $(<2 \mathrm{~cm})$, the transurethral resection is the treatment of choice although it presents a minor risk of incomplete surgery with need of a second surgery. In our case there was no tumor recurrence after transurethral surgery and at 6 months followup no recurrence of symptoms.

Extra vesical and large tumors need open surgery or a partial cystectomy based on their size (9). The rate of recurrence is low and this tumor has a good prognosis.

\section{Conclusion}

According to the non-specificity of the imaging, patient's age and clinical manifestations, differential diagnosis of atypical leiomyoma with bladder cancer is recommended. The diagnosis was confirmed only with histopathologic findings.

\section{REFERENCES}

1. Wong-You-Cheong JJ, Woodward PJ, Manning MA, Sesterhenn IA. From the archives of the AFIP: neoplasms of the urinary bladder: radiologic-pathologic correlation. Radiographics. 2006; 26:553-580.

2. Goktug GH, Ozturk U, Sener NC, et al. Transurethral resection of a bladder leiomyoma: a case report. Can Urol Assoc J. 2014; 8:E111-E113.

3. Greco A, Baima C, Piana P. Leiomyoma of the bladder. Report of a case and review of the literature. Minerva Urol Nefrol. 1999; 51:33-8.

4. Kretschmer HL Leiomyoma of the bladder with a report of a case and a review of the literature. J Urol. 1931; 26:575-90.

5. Kalathia J, Agrawal S, Chipde SS, Agrawal R. Total endoscopic management of a large bladder leiomyoma. Urol Ann. 2015; 7:527-9.

6. Chatterjee S, Baksi A, Kumar S. Dyspareunia due to leiomyoma of the urinary bladder: an unusual presentation of a rare bladder neoplasm. Hellenic J Surg. 2014; 86:34-36.

7. Cornella JL, Larson TR, Lee RA, et al. Leiomyoma of the female urethra and bladder: report of twenty-three patients and review of the literature. Am J Obstet Gynecol. 1997; 176:1278-1285.

8. Wu S. Imaging findings of atypical leiomyoma of the urinary bladder simulating bladder cancer: a case report and literature review. Med Ultrason. 2013, 15:161-163.

9. Jeschke K, Wakoning J, Winzely M, Henning K. Laparoscopic partial cystectomy for leiomyoma of the bladder wall. J Urol. 2002; 165:2115-6.

\section{Correspondence}

Mojtaba Ameli, MD (Corresponding Author)

mojtaba.ameli@gmail.com

Fellowship of Endourology

Iran University of Medical Science, Hasheminejad Kidney Center

Valinejad Avenue, Tehran, 1969714713 - Iran

Mina Rahmandoost

Gonabad University of Medical Sciences, Gonabad, Iran 